\title{
Propuesta de acceso múltiple al espectro en redes inalámbricas cognitivas
}

\section{Proposal for multiple spectrum access in cognitive wireless networks}

\author{
César Augusto Hernández Suárez ${ }^{(\mathbb{D}}$, Jorge Rodríguez García ${ }^{2}{ }^{(\mathbb{D}}$, Harold Peña Medina ${ }^{3}$
}

Cómo citar: Hernández S., C.A., Rodríguez G., J.A. y Peña M., H. (2020). Propuesta de acceso múltiple al espectro en redes inalámbricas cognitivas. Tecnura, 24(63) 13-25. DOI: https://doi.org/10.14483/22487638.15243

Fecha de de recepción: 3 de septiembre de 2018

\section{RESUMEN}

Contexto: El acceso a las redes inalámbricas es un proceso en el que intervienen múltiples agentes, por lo que resulta práctico e interesante realizar un análisis multiusuario.

Objetivo: Evaluar un algoritmo de asignación espectral en redes inalámbricas cognitivas que permita simular entornos con múltiples usuarios secundarios que acceden al espectro de forma simultánea.

Metodología: La asignación espectral se realiza mediante la evaluación, para cada usuario secundario, de las rutas espectrales encontradas con una adaptación del algoritmo colonia de abejas artificiales. La validación se da a partir de datos de ocupación espectral reales capturados en una campaña de medición.

Resultados: A partir de los resultados de simulaciones de la red cognitiva con múltiples usuarios secundarios, a través del algoritmo propuesto, se evalúan sus parámetros de interés del rendimiento, como la población de abejas para el tiempo de evaluación, número de handoffs totales y tiempo de ejecución.Conclusiones: El rendimiento del
Fecha de de aceptación: 14 de febrero de 2019

algoritmo es aceptable con respecto al tiempo de ejecución y el número de handoffs totales; sin embargo, se encuentra unatendencia al incremento de handoffs espectrales, conforme aumenta el número de usuarios secundarios en la red de radio cognitiva, por lo que un número elevado de estos disminuye el rendimiento de la red.

Palabras clave: acceso multiusuario; asignación espectral; espectro radioeléctrico; inteligencia de enjambre; radio cognitiva; redes inalámbricas.

\section{ABSTRACT}

Context: Access to wireless networks is a process in which multiple agents intervene, so it is practical and interesting to perform a multi-user analysis.

Objective: To evaluate a spectral allocation algorithm in cognitive wireless networks that allows simulating environments with multiple secondary users that access the spectrum simultaneously.

Methodology: The spectral assignment is performed by evaluating for each secondary user the spectral routes found with an adaptation of the Colony algorithm of Artificial Bees. Validation is performed

1 Ingeniero electrónico, doctor en Ingeniería. Docente e investigador de la Universidad Distrital Francisco José de Caldas. Bogotá, Colombia. Contacto: cahernandezs@udistrital.edu.co ORCID: https://orcid.org/0000-0001-9409-8341

2 Ingeniero eléctrico. Ingeniero de UL de Colombia. Bogotá, Colombia. Contacto: jarodriguezg@ul.com

ORCID: https://orcid.org/0000-0001-9482-0154

3 Ingeniero eléctrico. Profesional de Laboratorios de la Universidad de los Andes. Bogotá, Colombia. Contacto: h.pena@uniandes.edu.co ORCID: https://orcid.org/0000-0003-3766-4583 
based on actual spectral occupation data captured in a measurement campaign.

Results: Based on the results of simulations of the cognitive network with multiple secondary users using the proposed algorithm, parameters of interest of the algorithm's performance are evaluated, such as the bee population for the evaluation time, number of total handoffs and execution time.

Conclusions: The performance of the algorithm is acceptable with respect to the execution time and the number of total handoffs, however, there is a tenden cy to increase spectral handoffs as the number of secondary users in the cognitive radio network increases, so a high number of secondary users decreases network performance.

Keywords: multi-user access; spectral allocation, radio spectrum; swarm intelligence; cognitive radio; wireless networks.

\section{INTRODUCCIÓN}

El desarrollo tecnológico de las comunicaciones inalámbricas en la época actual ha posibilitado un aumento drástico en el uso de dispositivos inalámbricos, razón por la cual algunas bandas de frecuencia del espectro radioeléctrico son ampliamente usadas (Nandini y Hariprasad, 2019; Mejía, 2017; Narváez, 2016). La radio cognitiva plantea usar de una manera eficiente el espectro, mediante la búsqueda y asignación de bandas de frecuencia disponibles a usuarios secundarios, siempre que el original (primario) no le esté dando uso. En el caso de que el usuario primario (UP) requiera de su respectiva banda de frecuencia, el secundario (US) se retira y da un salto a otra frecuencia (handoff espectral) en búsqueda de una nueva disponible; así se da continuidad a la transmisión tanto de usuarios primarios como secundarios (Ponce, 2019; Sánchez, 2016; Hernández, Páez y Giral, 2015; Bordón y Montejo, 2015; Pedraza, López y Salcedo, 2011).

Hay varios estudios y publicaciones en el área de radio cognitiva, como se muestra en Wang y Ray Liu (2011) y Ghosh, Das y Chatterjee (2014); sin embargo, la mayoría de ellos se ha enfocado en proponer y validar algoritmos para el acceso al espectro en ambientes de un solo usuario, lo cual se aleja un poco de la realidad al no considerar un acceso multiusuario (Shetty, Agbedanu y Ramachandran, 2011; Carrillo,Mathilde, Yoshimura y Bazzo, 2013; Casadevall y Perejil, 2016; Hernández, Pedraza, Páez y Rodríguez, 2015; Ordoñez, 2016). Uno de los pocos trabajos que presenta un análisis multiusuario para redes de radio cognitiva es el de Liu, Nasser y Hassanein (2013), en el que se evalúa el rendimiento de dos algoritmos de asignación espectral en redes multiusuario: algoritmo genético y algoritmo heurístico. Los resultados, mediante el ajuste de los parámetros de la red cognitiva y el aumento el número de US, muestran que para niveles de tráfico bajo la asignación espectral es más eficiente el algoritmo genético, aunque pierda su funcionalidad en niveles de tráfico alto, donde dicho algoritmo conserva un buen rendimiento.

El presente artículo tiene por objetivo evaluar el desempeño de un algoritmo de asignación espectral para redes de radio cognitiva con un enfoque de acceso multiusuario simultáneo, basado en el algoritmo de colonia de abejas artificiales (ABC, por su sigla en inglés). El algoritmo propuesto se valida a través de datos de ocupación espectral reales, capturados en una campaña de medición espectral anterior, sobre la banda de frecuencia GSM (Pedraza, Forero y Páez, 2014). Las métricas de evaluación utilizadas son el número de handoffs es- 
pectrales totales, durante una transmisión de diez minutos. Posteriormente, se contrastan los resultados obtenidos con los de Liu, Nasser y Hassanein (2013), con el propósito de iniciar una evaluiciar una evaluación y análisis comparativo.

\section{METODOLOGÍA}

El punto de partida para desarrollar el algoritmo propuesto y su evaluación es obtener los datos de ocupación espectral obtenidos a partir de la monitorización del espectro. Luego, se les debe aplicar un tratamiento que se explicará a continuación, con el fin de que sea posible adaptar el algoritmo $A B C$ y simular una red de radio cognitiva con cinco usuarios secundarios (US) que intentan transmitir simultáneamente.

Se adelantan múltiples simulaciones con variación de tamaño de la población de abejas, para así estudiar su influencia y encontrar un valor con el que tenga mejor rendimiento la red en general. Cuando se identifique dicho nivel de población, se estudian los cambios de otros parámetros importantes a tener en cuenta del algoritmo, con el fin de establecer nociones claras del comportamiento real de la red.

\section{Datos de ocupación espectral}

En campañas de mediciones previas, se obtuvieron los datos de ocupación espectral mediante la técnica de detección de energía, a partir de la cual se construyó una matriz de potencias. Posteriormente, se definió, por medio de la probabilidad de falsa alarma, el umbral de decisión para determinar la ocupación o disponibilidad de cada canal de frecuencia monitorizado, definiendo la matriz de disponibilidad.

Después de un análisis estadístico se categorizan dos niveles de ocupación espectral, los cuales se denominan tráfico alto y tráfico bajo, que se pueden ver como el comportamiento del espectro radioeléctrico en una condición de una gran cantidad de usuarios primarios intentando comunicarse (tráfico alto), y encondición de una baja cantidad de usuarios primariosintentando comunicarse (tráfico bajo).

Adicionalmente, el algoritmo propuesto requiere que se dividan los datos de ocupación espectral en dos trazas de igual tamaño: la primera se utiliza paraentrenar el algoritmo, de lo cual se obtienen alternativas de bandas de frecuencia libre (denominadas rutas espectrales); a la segunda se recurre para simular una red de radio cognitiva en la que cinco US intentarán establecer comunicación a través de las rutas espectrales halladas anteriormente.

\section{Algoritmo ABC propuesto}

Para llevar a cabo la etapa de entrenamiento del algoritmo propuesto se investigó en la literatura un método que tuviera las características de búsqueda, selección y versatilidad, junto con un tiempo de solución no tan elevado. Los aspectos anteriores son necesarios ya que, dentro de una red de comunicaciones real, la disponibilidad de las bandas de frecuencia puede presentar cambios abruptos en pequeños intervalos de tiempo (Peng, Jain y Dehuri, 2009).

En ese sentido, se escoge el algoritmo artificial bee colony $(A B C)$, el cual es de inteligencia de enjambre que simula el comportamiento de una colonia de abejas durante la búsqueda de alimento. Como se evidencia más adelante, $\mathrm{ABC}$ se adapta a las necesidades requeridas debido a que la búsqueda y selección de fuentes de alimento por las abejas es un proceso cíclico que, aunque tiene matices tanto aleatorios como probabilísticos, su solución final 
mejora notablemente con cada ciclo. Esto se logra con el uso de pocas ecuaciones prácticas, por lo que también queda cubierto el requerimiento de un tiempo de ejecución bajo.

Ahora, se da una explicación del algoritmo $A B C$ para luego indicar cómo se adapta en la búsqueda y determinación de las rutas espectrales que se usarán en la simulación de una red inalámbrica cognitiva con varios US simultáneos.

El algoritmo $\mathrm{ABC}$ concibe tres grupos de abejas en el proceso de búsqueda de fuentes de alimento: empleadas, observadoras y exploradoras, cada una de ellas tiene una función específica. Las empleadas explotan varias fuentes de alimento y comunican el potencial de cada fuente encontrada a las observadoras, quienes seleccionan las mejores fuentes de alimento basándose en su potencial; por último, las exploradoras encuentran nuevas fuentes de alimento para su explotación por parte de las empleadas (Karaboga y Basturk, 2007). Este proceso se repite cíclicamentecuando las observadoras seleccionan las mejores fuentes de alimento encontradas, las cuales corresponden a las posibles soluciones de un problema específico.

El algoritmo $A B C$ termina cuando se cumple un determinado número de ciclos definido inicialmente o cuando se encuentra una solución aceptable.

Para mostrar las ecuaciones usadas en el algoritmo $A B C$ se deben definir sus parámetros de entrada:

-Tamaño de la población de abejas: SN.

-Valores mínimo y máximo en el que se presume estará la solución:

-Número de ciclos máximo: MCN.

-Se puede definir un límite de ciclos en el que se conserva una fuente de alimento; si no mejora después de estos ciclos, será reemplazada por una nueva: L.

En un primer momento, se genera un número SN de soluciones iniciales $x i$ con la ecuación (1):

$$
\begin{gathered}
x i=x_{\min }+\operatorname{rand}(0,1)\left(x_{\max }-x_{\min }\right) \\
i \in[1,2,3,4 \ldots S N]
\end{gathered}
$$

Posteriormente, se evalúa el potencial si de cada fuente de alimento xi encontrada con la ecuación característica del problema a solucionar y se halla un número $\mathrm{SN}$ de nuevas fuentes de alimento cercanas yi con la ecuación (2). De igual forma, se evalúa el potencial si de las nuevas fuentes de alimento encontradas yi y se selecciona la mejor opción entre las posibles yi y xi, este valor es reemplazado en xi.

$$
\begin{gathered}
y i=x j+\varnothing j(x j-x k) \\
j \neq k
\end{gathered}
$$

\section{Øj número real aleatorio entre $[-1,1]$}

Ahora, la selección de fuentes de alimento por parte de las abejas observadoras se realiza teniendo en cuenta la probabilidad $P i$ para cada fuente de alimento xi que se calcula con la ecuación (3). El proceso se repite a partir de la ecuación (2), generando nuevas fuentes de alimento cercanas y seleccionando las mejores. Se repite un número de ciclos $\mathrm{MCN}$ o hasta que se encuentre una solución aceptable para el problema en cuestión.

$$
P_{j}=\frac{S_{i}}{\sum_{\mathrm{n}=1}^{\mathrm{SN}} \mathrm{S}_{\mathrm{n}}}
$$


El procedimiento del algoritmo $\mathrm{ABC}$ descrito se adapta para encontrar varias rutas espectrales libres quelos US tendrán como referencia para establecer co-municación. Para dicho fin, se usará la primera traza de ocupación espectral para encontrar tales rutas espectrales. En ella, las soluciones xi serán los intervalos de tiempo en la que una banda de frecuencia se encuentre libre, y el potencial si de dicho intervalo será su tiempo de disponibilidad.

El procedimiento del algoritmo $A B C$ descrito se adapta para encontrar varias rutas espectrales libres que los US tendrán como referencia para establecer comunicación. Para dicho fin, se usará la primera traza de ocupación espectral para encontrar tales rutas espectrales. En ella, las soluciones $x i$ serán los intervalos de tiempo en la que una banda de frecuencia se encuentre libre, y el potencial si de dicho intervalo será su tiempo de disponibilidad.

De esta manera se busca seleccionar los intervalos $x i$ que tengan mayor tiempo de disponibilidad y añadirlos uno a uno a la ruta espectral. La búsqueda de una ruta espectral se detiene cuando se tengan intervalos $x i$ definidos para toda la extensión de la traza. La etapa de entrenamiento termina cuando se encuentre el número de rutas espectrales requeridas definidas inicialmente por quien realice la simulación.

Al terminar la etapa de entrenamiento se comienza la etapa de evaluación, en la que se usa la segunda traza de ocupación espectral y se busca asignar a cada US una banda de frecuencia libre para toda la extensión de tiempo de la traza. Para esto, se evalúa la disponibilidad de las bandas de frecuencia indicadas por las rutas espectrales encontradas en la etapa de entrenamiento, a partir del siguiente procedimiento.
En el inicio de la traza se evalúa si la banda de frecuencia indicada por la ruta espectral se encuentra ocupada para ese instante de tiempo; si está ocupada, se procede a evaluar la banda de frecuencia que indica la siguiente ruta espectral encontrad hasta hallar una disponible. Cuando se localice una banda de frecuencia disponible, el US establece comunicación a través de ella, y se le asigna otra banda de frecuencia libre al siguiente US de manera análoga. Cuando todos los US tengan asignada una banda de frecuencia libre, se aumenta el instante de tiempo de evaluación y se repite la asignación de bandas de frecuencia que se describió en el párrafo anterior. De esta forma se evalúan las rutas espectrales obtenidas a lo largo de toda la traza guardando la evolución de los handoffs espectrales totales y fallidos que serán el resultado de la simulación. Para tal efecto, se asume un handoff fallido cuando en medio de la simulación se requiere cambiar de banda de frecuencia y al evaluar la banda de la siguiente ruta espectral se encuentra ocupada; mientras que un handoff exitoso se da cuando al evaluar la banda de la siguiente ruta espectral se encuentra disponible. Los handoffs totales se toman como la suma de los exitosos y los fallidos.

Experimentación: Para realizar la validación y evaluación del algoritmo propuesto, en la presente investigación se adelanta un conjunto de simulaciones sobre un software para redes de radio cognitiva desarrollado previamente (Hernández, Páez y Giral, 2016). Dicho software fundamenta su funcionamiento en datos de ocupación espectral reales capturados en la banda de frecuencia GSM en una campaña de medición anterior, llevada a cabo en la ciudad de Bogotá. Lo anterior permite que la validación y evaluación se acerquen un poco más a la realidad en cuanto que su desempeño está medido sobre un patrón de comportamiento 
real de usuarios primarios. Adicionalmente, para tener un estadístico significativo se decidió adelantar diez simulaciones de cada experimento y tomar el valor promedio de los resultados obtenidos.

\section{Métricas de evaluación}

Para evaluar el algoritmo propuesto se tendrán en cuenta tres criterios: tamaño de población de colonia de abejas, número de handoffs totales por usuario y tiempo de ejecución. En la sección de resultados y conclusiones se discute sobre la forma de aplicar tales criterios.

\section{RESULTADOS}

Los resultados obtenidos se organizan en tres secciones. La primera muestra el análisis realizado para determinar el tamaño de población con mejores resultados. La segunda utiliza el resultado anterior para determinar las tasas de handoff espectral correspondientes a cada uno de los cinco usuarios secundarios (US) que acceden al espectro de manera simultánea, tanto en tráfico alto como en trá- fico bajo. La tercera sección analiza los tiempos de ejecución del algoritmo propuesto en ambos niveles de tráfico, durante los diez minutos de transmisión simultánea de los cinco US. La cuarta sección presenta un análisis comparativo con otro trabajo relacionado.

\section{Población de abejas}

Según lo planteado en las secciones anteriores, en la tabla 1. se muestran los resultados promedio de cinco simulaciones, para hando$\mathrm{ffs}$ totales (tráfico alto y bajo), en una red de radio cognitiva con cinco US y para cada nivel de población.

Al obtener los resultados anteriores se procede a calcular una calificación del rendimiento por usuario para cada nivel de población, teniendo como referencia el número de handoffs totales: se compara el número de handoffs por US, con el valor mínimo obtenido entre todas las poblaciones; así se alcanza una calificación que puede tener un valor menor o igual a 1; donde 1 indica la población con

Tabla 1. Promedio de handoffs totales para cinco niveles de población y dos niveles de tráfico

\begin{tabular}{llllll}
\hline \multicolumn{7}{l}{ Promedio por población handoffs totales tráfico alto } \\
\hline SU/Población & 100 & 200 & 300 & 400 & 500 \\
\hline SU1 & 519 & 450 & 465 & 437 & 449 \\
\hline SU2 & 499 & 474 & 448 & 449 & 473 \\
\hline SU3 & 524 & 532 & 494 & 483 & 505 \\
\hline SU4 & 576 & 582 & 511 & 526 & 550 \\
\hline SU5 & 573 & 609 & 589 & 529 & 543 \\
\hline Promedio por población handoffs totales tráfico bajo & & \\
\hline SU/Población & 100 & 200 & 300 & 400 & 500 \\
\hline SU1 & 328 & 313 & 317 & 326 & 324 \\
\hline SU2 & 283 & 358 & 335 & 370 & 315 \\
\hline SU3 & 395 & 378 & 351 & 366 & 372 \\
\hline SU4 & 420 & 397 & 362 & 361 & 353 \\
\hline SU5 & 422 & 423 & 404 & 392 & 395 \\
\hline
\end{tabular}

Fuente: elaboración propia. 
la que se logra el menor número de handoffs totales para su correspondiente usuario, y una calificación menor a esta cifra desciende conforme el número de handoffs aumenta.

El procedimiento anterior se realiza para cada US y para los dos niveles de tráfico. Por último se obtiene un valor promedio de las calificaciones para los dos niveles de tráfico, el cual será con el que se defina el nivel de población que cuenta con un mayor rendimiento para un tiempo de evaluación de diez minutos.
El procedimiento descrito se detalla en la tabla 2, donde la población con mejor rendimiento es 400 al obtener la calificación total más alta $(0,963)$.

El estudio previo para determinar la población más eficiente mostrado en las tablas 1 y 2 es un paso adicional característico del algoritmo propuesto, y se debe realizar siempre que se quiera encontrar el mayor rendimiento de la red inalámbrica cognitiva. Se debe tener en cuenta que la mejor población encontrada es válida para el tiempo de

Tabla 2. Calificación de cada nivel de población con base en el número de handoffs totales

\begin{tabular}{lccccl}
\hline \multicolumn{6}{l}{ Calificación por población handoffs totales tráfico alto } \\
\hline SU/población & 100 & 200 & 300 & 400 & 500 \\
\hline SU1 & 0,842 & 0,971 & 0,940 & 1,000 & 0,973 \\
\hline SU2 & 0,898 & 0,945 & 1,000 & 0,998 & 0,947 \\
\hline SU3 & 0,922 & 0,908 & 0,978 & 1,000 & 0,956 \\
\hline SU4 & 0,887 & 0,878 & 1,000 & 0,971 & 0,929 \\
\hline SU5 & 0,923 & 0,869 & 0,898 & 1,000 & 0,974 \\
\hline Calificación por población handoffs totales tráfico bajo & & \\
\hline SU/población & 100 & 200 & 300 & 400 & 500 \\
\hline SU1 & 0,954 & 1,000 & 0,987 & 0,960 & 0,966 \\
\hline SU2 & 1,000 & 0,791 & 0,845 & 0,765 & 0,898 \\
\hline SU3 & 0,889 & 0,929 & 1,000 & 0,959 & 0,944 \\
\hline SU4 & 0,840 & 0,889 & 0,975 & 0,978 & 1,000 \\
\hline SU5 & 0,929 & 0,927 & 0,970 & 1,000 & 0,992 \\
\hline Calificación promedio por población & & & \\
\hline TOTAL & 0,908 & 0,911 & 0,959 & 0,963 & 0,958 \\
\hline
\end{tabular}

Fuente: elaboración propia.

evaluación de estudio y puede sufrir variaciones si se cambia el tiempo de evaluación; es decir, en el estudio se encontró que la mejor población con la que se tiene un mayor rendimiento es de 400 abejas artificiales para un tiempo de evaluación de 10 minutos, si se cambia el tiempo de evaluación, se puede encontrar con que una población diferente a 400 es con la que se presenta el mayor rendimiento.

\section{Tasa de handoff}

Con la población de mejor rendimiento definida, se realizaron diez simulaciones para 
evaluar el desempeño del algoritmo propuesto de acuerdo con la tasa de handoff, cuyos resultados se muestran en las tablas 3 y 4 .

Con el valor promedio se puede identificar una tendencia del comportamiento de los handoffs totales. Principalmente, se encuentra que hay un mayor número de handoffs totales para un nivel de tráfico alto, para el que se tienen valores desde 449 hasta 582 handoffs, mientras que para el nivel de tráfico bajo se tienen valores desde 310 hasta 408 , lo cual era de esperarse debido a que al tener un mayor nivel de ocupación se requiere cambiar, con mayor continuidad, de banda de frecuencia. Además, conforme aumenta el orden del US, se tiende a aumentar el número de handoffs, es decir, para el primer US se da el menor número de handoffs y para el quinto US se da el mayor número de handoffs. Lo anterior se debe a que para los primeros $\mathrm{SU}$ se usan las primeras rutas espectrales obtenidas en la etapa de entrenamien- to, las cuales generalmente tienen un tiempo de disponibilidad mayor, mientras que para los últimos US se debe recurrir a las últimas rutas espectrales, con tiempo de disponibilidad menor, y además deben ajustarse a los nuevos cambios en la traza de disponibilidad generada por los primeros US.

Con respecto a los handoffs totales obtenidos en las tablas 3 y 4 , se identifica una tendencia al incremento en el número de handoffs, conforme aumenta laposición del US al hacer una revisión de los handoffs promedio. Aunque en algunas simulaciones individuales no se presenta claramente dicha tendencia; esto se puede atribuir a cómo se construyen las rutas espectrales, en donde al usar una adaptación de las ecuaciones (1) y (2) en las que existe un factor aleatorio, se traduce en que no necesariamente las primeras rutas espectrales halladas son las que tienen los mejores tiempos de disponibilidad, ya que dependen de los tramos disponibles hallados en la búsqueda aleatoria.

Tabla 3. Recuento de handoffs totales para tráfico alto, por cada simulación para una población de 400

\begin{tabular}{lccccccccccc}
\hline \multirow{2}{*}{ Usuario } & \multicolumn{10}{c}{ Handoffs totales para tráfico alto por simulación } & \multirow{2}{*}{ Promedio } \\
\cline { 2 - 11 } & 1 & 2 & 3 & 4 & 5 & 6 & 7 & 8 & 9 & 10 & \\
\hline SU 1 & 471 & 536 & 463 & 409 & 455 & 391 & 414 & 444 & 514 & 394 & 449 \\
\hline SU 2 & 444 & 449 & 528 & 443 & 504 & 436 & 473 & 498 & 378 & 447 & 460 \\
\hline SU 3 & 559 & 580 & 456 & 525 & 446 & 525 & 436 & 436 & 493 & 515 & 497 \\
\hline SU 4 & 607 & 510 & 525 & 588 & 523 & 466 & 494 & 580 & 528 & 498 & 532 \\
\hline SU 5 & 653 & 604 & 694 & 502 & 554 & 608 & 541 & 614 & 543 & 507 & 582 \\
\hline
\end{tabular}

Fuente: elaboración propia.

Tabla 4. Recuento de handoffs totales para tráfico bajo, por cada simulación para una población de 400

\begin{tabular}{lccccccccccc}
\hline \multirow{2}{*}{ Usuario } & \multicolumn{10}{c}{ Handoffs totales para tráfico alto por simulación } & \multirow{2}{*}{ Promedio } \\
\cline { 2 - 11 } & 1 & 2 & 3 & 4 & 5 & 6 & 7 & 8 & 9 & 10 & \\
\hline SU 1 & 280 & 348 & 262 & 336 & 361 & 325 & 330 & 253 & 240 & 362 & 310 \\
\hline SU 2 & 354 & 367 & 333 & 433 & 298 & 332 & 340 & 396 & 388 & 390 & 363 \\
\hline SU 3 & 304 & 357 & 446 & 383 & 351 & 362 & 380 & 419 & 374 & 342 & 372 \\
\hline SU 4 & 324 & 377 & 343 & 408 & 309 & 377 & 352 & 421 & 333 & 403 & 365 \\
\hline SU 5 & 335 & 337 & 471 & 363 & 369 & 526 & 394 & 445 & 457 & 379 & 408 \\
\hline
\end{tabular}

Fuente: elaboración propia. 
Por otro lado, la tendencia encontrada en el promedio de los handoffs está condicionada por el hecho de que los primeros US van a ocupar primero las bandas de frecuencia libres, por lo que los últimos deben realizar una mayor búsqueda.

\section{Tiempos de ejecución}

Ahora, con la misma población de 400, se realizaron 10 simulaciones para estimar el tiempo de ejecución del algoritmo propuesto tanto en tráfico alto como bajo, cuyos resultados se muestran en las tablas 5 y 6 .

En cuanto al tiempo de ejecución, este es mayor cuando se evalúa con una traza de disponibilidad de tráfico alto, para el que se tiene en promedio de ejecución de 132,2 segundos; mientras que para tráfico se tiene un promedio de 60,1 s. Esta tendencia se puede esperar debido a que un mayor nivel de ocupación se traduce en un mayor tiempo de búsqueda de las rutas espectrales requeridas.

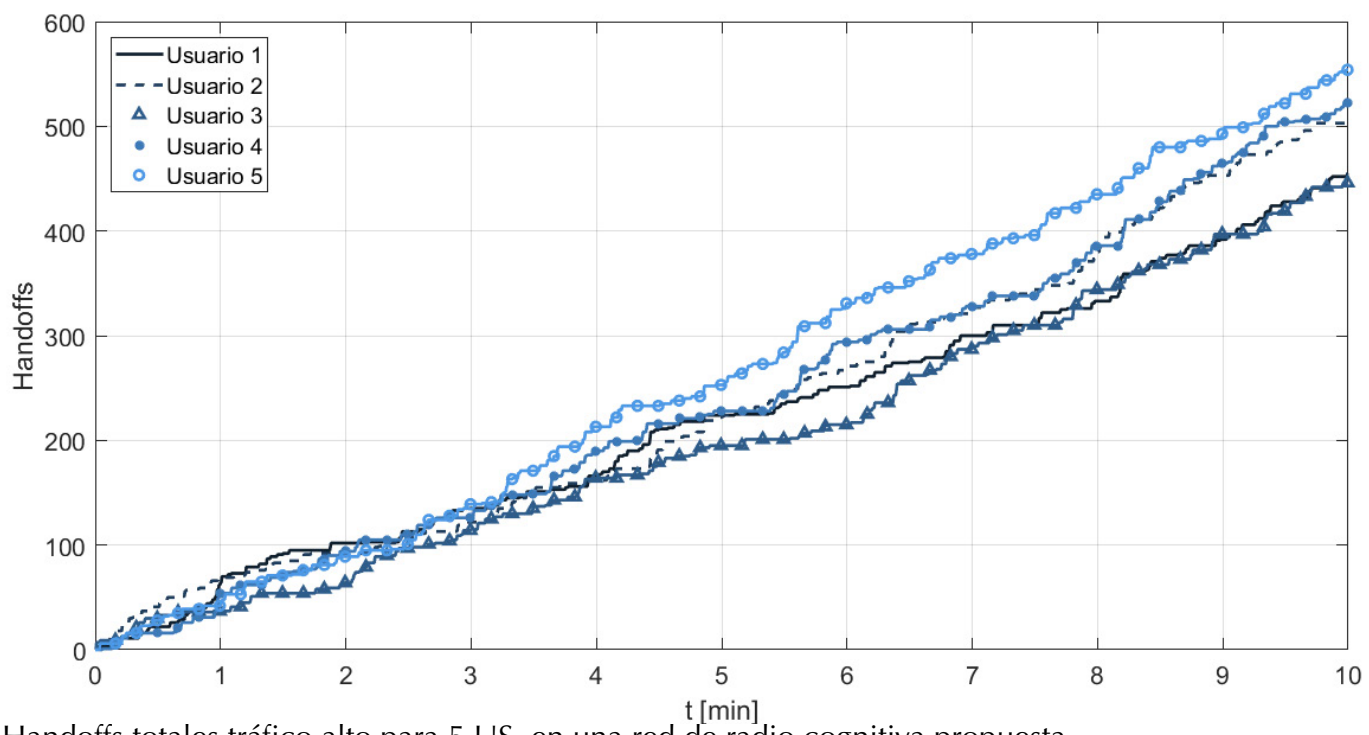

Figura 1. Handoffs totales tráfico alto para 5 US, en una red de radio cognitiva propuesta Fuente: elaboración propia.

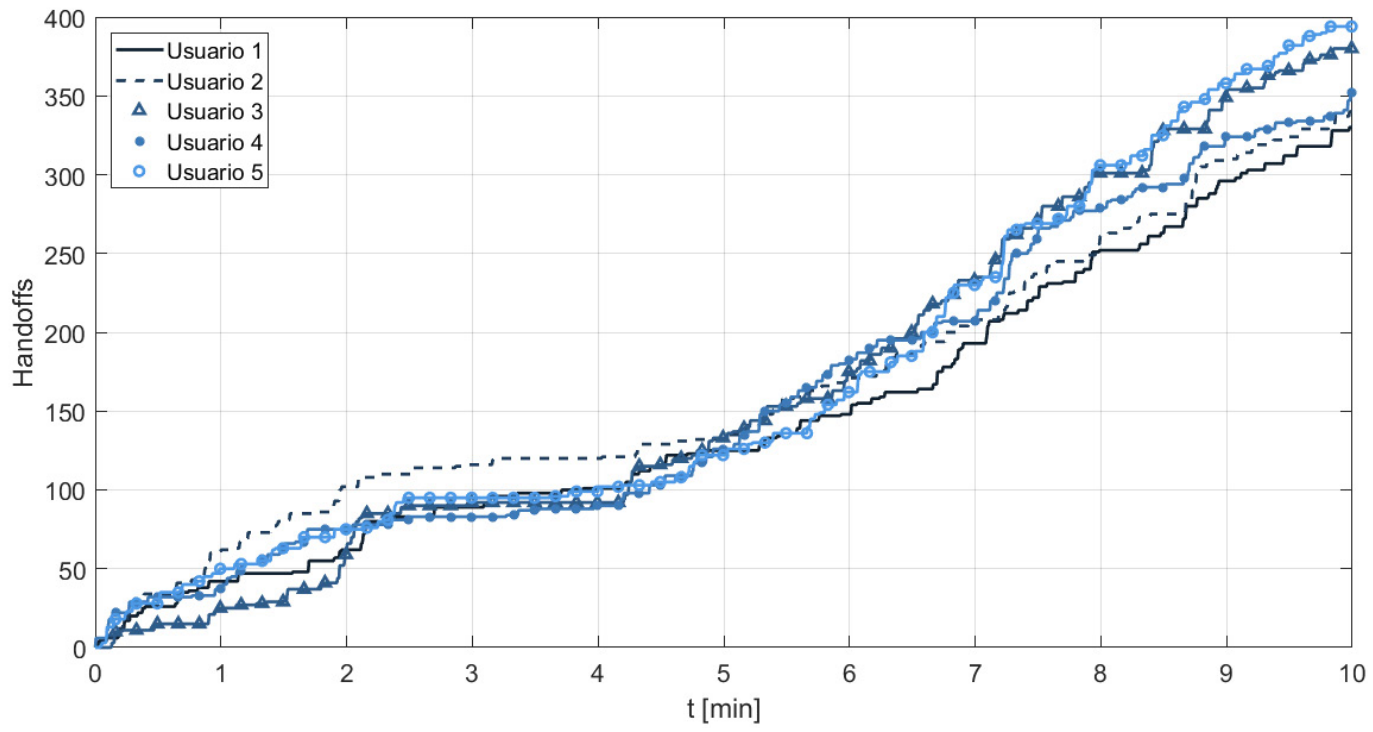

Figura 2. Handoffs totales tráfico bajo para 5 US, en una red de radio cognitiva propuesta Fuente: elaboración propia. 
Los tiempos de ejecución encontrados en las tablas 5 y 6 . no son excesivos si se comparan con el tiempo de evaluación; para la traza de nivel de tráfico alto se obtuvo en promedio de ejecución 132,2 s, lo que corresponde a $22,03 \%$ del tiempo de evaluación (600 s), y para la traza de nivel de tráfico bajo se obtuvo en promedio un tiempo de ejecución de 60,1s,

Tabla 5. Recuento de tiempo de ejecución para tráfico alto, por cada simulación, para una población de 400

\begin{tabular}{|c|c|c|c|c|c|c|c|c|c|c|c|}
\hline \multirow{2}{*}{ Usuario } & \multicolumn{10}{|c|}{ Tiempo de ejecución para tráfico alto por simulación [s] } & \multirow{2}{*}{ Promedio } \\
\hline & 1 & 2 & 3 & 4 & 5 & 6 & 7 & 8 & 9 & 10 & \\
\hline \multicolumn{12}{|l|}{ SU 1} \\
\hline \multicolumn{12}{|l|}{ SU 2} \\
\hline SU 3 & 108,8 & 143,9 & 127,4 & 131,9 & 138,7 & 138,6 & 150,9 & 122,2 & 128,4 & 131,0 & 132,2 \\
\hline \multicolumn{12}{|l|}{ SU 4} \\
\hline SU 5 & & & & & & & & & & & \\
\hline
\end{tabular}

Fuente: elaboración propia.

Tabla 6. Recuento de tiempo de ejecución para tráfico bajo, por cada simulación, para una población de 400

\begin{tabular}{|c|c|c|c|c|c|c|c|c|c|c|c|}
\hline \multirow{2}{*}{ Usuario } & \multicolumn{10}{|c|}{ Tiempo de ejecución para tráfico bajo por simulación [s] } & \multirow{2}{*}{ Promedio } \\
\hline & 1 & 2 & 3 & 4 & 5 & 6 & 7 & 8 & 9 & 10 & \\
\hline SU 1 & \multirow{5}{*}{47,7} & \multirow{5}{*}{69,3} & \multirow{5}{*}{58,3} & \multirow{5}{*}{62,3} & \multirow{5}{*}{62,3} & \multirow{5}{*}{64,2} & \multirow{5}{*}{54,6} & \multirow{5}{*}{59,8} & \multirow{5}{*}{57,9} & \multirow{5}{*}{64,5} & \multirow{5}{*}{60,1} \\
\hline SU 2 & & & & & & & & & & & \\
\hline SU 3 & & & & & & & & & & & \\
\hline SU 4 & & & & & & & & & & & \\
\hline SU 5 & & & & & & & & & & & \\
\hline
\end{tabular}

Fuente: elaboración propia.

lo que corresponde a 10,01 \% del tiempo de evaluación (600 s). Son cifras aceptables, si se piensa en una posible aplicación real en el futuro en la que se necesitan tiempos de ejecución menores que el tiempo de evaluación.

Evaluación comparativa Con el ánimo de plantear una discusión y demostrar el aporte del presente artículo, a continuación se aborda el trabajo realizado por Liu, Nasser y Hassanein (2013), quienes desarrollan un algoritmo genético para optimizar la asignación espectral en redes cognitivas con múltiples usuarios secundarios. También, utilizan una función objetivo en la que se relacionan parámetros de la red cognitiva como rendimiento de la red e ingresos del operador de red para cada usuario secundario, además de los parámetros propios de un algoritmo genético, como tasa de mutación, tasa de cruce, tamaño de la población de individuos y número de generaciones. Los autores se centran en las características cualitativas de los resultados obtenidos con el fin de evidenciar la posibilidad de tener enfoques diferentes que se le pueden dar a la misma tarea del desarroIlo de algoritmos de asignación espectral; en vez de realizar un análisis cuantitativo completo debido a que no se especifican tiempos de evaluación y no se tiene en cuenta la evolución de los handoffs espectrales en los algoritmos propuestos.

El algoritmo genético presenta un buen rendimiento con respecto a velocidad y cumple 
su objetivo de optimizar el rendimiento de la red; sin embargo, este último disminuye drásticamente conforme aumenta el nivel de tráfico de la red hasta no poder ejecutar la asignación espectral dentro de una red cognitiva muy congestionada. En la misma publicación, se presenta el funcionamiento de un algoritmo heurístico y se compara su rendimiento con el algoritmo genético; aquí se encuentra que este último posee un buen rendimiento sin importar que el nivel de congestión de la red aumente, con lo cual queda claro que, para niveles de congestión bajo, el algoritmo genético ofrece mejores resultados. Aunque, en ninguno de los dos algoritmos se presentan datos que permitan comparar cuantitativamente los resultados obtenidos.

En cuanto a las diferencias en el método de simulación, en este se asigna un número de bandas del espectro disponible $M y$, para simular diferentes niveles de tráfico, se cambia el número de US, así: para simular una red congestionada asignan $\mathrm{SU}>\mathrm{M}$, y para simular una red no congestionada asignan $\mathrm{SU}<\mathrm{M}$; mientras que, en el presente artículo, los niveles de tráfico se diferencian por trazas generadas a partir de campañas de medición del espectro.

\section{CONCLUSIONES}

Las simulaciones del algoritmo propuesto con múltiples usuarios secundarios (US) simultáneos sirven como un primer acercamiento al estudio de la implementación de las redes inalámbricas cognitivas reales; brindan criterios de selección del tiempo de evaluación y del número de US máximos permitidos con el que se buscará un buen rendimiento sin handoffs totales excesivos y sin tiempos de ejecución del algoritmo excesivamente elevados.

\section{FINANCIAMIENTO}

El presente trabajo es el resultado de un proyecto de investigación financiado por el Centro de Investigaciones y Desarrollo Científico de la Universidad Distrital Francisco José de Caldas.

\section{REFERENCIAS}

Bordón L., R. y Montejo S., S. (2015). La radio cognitiva y su impacto en el uso eficiente del espectro de radio. Revista de Ingeniería Electrónica, Automática y Comunicaciones, 36(1), 42-55.

Carrillo, D., Mathilde, F., Yoshimura, R. y Bazzo, J. (2013). Red Experimental Cognitiva: Algoritmos y Resultados. En IEEE Colombian Conference on Communications and Computing (COLCOM) (pp. 1-5). Medellín. https://doi.org/10.1109/ ColComCon.2013.6564839

Casadevall Palacio, F. y Perejil Sabaté, I. (2016). Implementación de terminales de radio cognitiva en la banda de TV. Barcelona: Escola Tècnica Superior d’Enginyeria de Telecomunicació de Barcelona.

Ghosh, G., Das, P. y Chatterjee, S. (2014). Cognitive Radio and Dynamic Spectrum Access - A Study. International Journal of Next-Generation Networks, 6(1), 43-60. https://doi.org/10.5121/ ijngn.2014.6104

Hernández, C.A., Páez, I.P. y Giral, D.A. (2016). Modelo adaptativo multivariable de handoff espectral para incrementar el desempeño en redes de 
radio cognitiva. Bogotá: Universidad Distrital Francisco José de Caldas.

Hernández, C., Pedraza, L., Páez, I. y Rodríguez, E. (2015). Análisis de la movilidad espectral en redes de radio cognitiva. Información Tecnológica, 26(6). https://doi.org/10.4067/S071807642015000600018

Karaboga, D. y Basturk, B. (2007). Artificial Bee Colony (ABC) Optimization Algorithm for Solving Constrained Optimization Problems. En P. Melin, O. Castillo, L.T. Aguilar, J. Kacprzyk y W. Pedrycz (eds.), Foundations of Fuzzy Logic and Soft Computing (pp. 789-798). Berlín, Alemania: Springer. https://doi. org/10.1007/978-3-540-72950-1_77

Liu, Z., Nasser, N. y Hassanein, H.S. (2013). Heuristic Based Dynamic Spectrum Assignment in Cognitive Radio Network. En International Conference on Computing, Management and Telecommunications (ComManTel) (pp. 105-110). Ho Chi Min, Vietnam.https://doi.org/10.1109/ ComManTel.2013.6482374

Mejía Ardila, A. F. (2017). Análisis de ocupación de canales para redes de Radio Cognitiva en el espectro de señales GSM durante un periodo de seis meses. [Tesis de maestría]. Facultad de Ingeniería, Universidad Nacional de Colombia. Bogotá.

Nandini, K.S. y Hariprasad, S.A. (2019). Optimal Spectrum Sensor Assignment in Multi-channel Multi-user Cognitive Radio Networks. Journal of Telecommunications and Information Technology, 8(4), 88-96. https://doi.org/10.26636/ jtit.2018.124017
Narváez Romero, F.R. (2016). Diseño de un detector de energía para señales de radio FM en radio cognitiva. Loja: Universidad Nacional de Loja.

Ordoñez, J.C. (2016). Caracterización de usuarios primarios para la implementación de un modelo predictor para la toma de decisiones en redes inalámbricas de radio cognitiva. Bogotá: Universidad Distrital Francisco José de Caldas.

Pedraza, L.F., Forero, F. y Páez, I.P. (2014). Evaluación de ocupación del espectro radioeléctrico en Bogotá-Colombia. Ingeniería y Ciencia, 10(19), 127-143. https://doi.org/10.17230/ingciencia.10.19.6

Pedraza, L., López, D. y Salcedo, O. (2011). Enrutamiento basado en el algoritmo de Dijkstra para una red de radio cognitiva. Tecnura, 15(30), 94-100.

Peng Lim, C., Jain, L. y Dehuri, S. (2009). Innovations in Swarm Intelligence. India: Springer-Verlag Berlin Heidelberg. https://doi.org/10.1007/978-3-64204225-6

Ponce Pinos, J.E. (2019). Evaluación de ocupación del espectro radioeléctrico y análisis de factibilidad de uso de radio cognitiva en la banda UHF (450-512 $\mathrm{MHz}$ ) Para su optimización en la ciudad de Riobamba. Riobamba: Escuela Superior Politécnica de Chimborazo.

Sánchez López, C. (2016). Estudio sobre la viabilidad de redes radio cognitivas en el Campus Nord de la Universidad Politécnica de Cataluña. Barcelona: I' Escola Tècnica d'Enginyeria de Telecomunicació de Barcelona. 
Shetty, S., Agbedanu, K. y Ramachandran, R. (2011). Opportunistic spectrum access in multi-user multi-channel cognitive radio networks. En 19th European Signal Processing Conference, EUSIPCO 2011 (pp. 1229-1233). Barcelona, España.Wang, B. y Ray Liu, K.J. (2011). Advances in Cognitive Radio Networks: A Survey. IEEE Journal of Selected Topics in Signal Processing, 5(1), 5-23. https:// doi.org/10.1109/JSTSP.2010.2093210

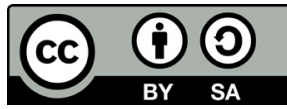

